LBNL-40176

Proceedings, International Workshop on Astro Hadron Physics,

'Hadrons in Dense Matter',

APCTP, Seoul, Korea, October 1997

\title{
DILEPTONS FROM DISORIENTED CHIRAL CONDENSATES
}

\author{
VOLKER KOCH, JØRGEN RANDRUP, XIN-NIAN WANG \\ Nuclear Science Division, Lawrence Berkeley National Laboratory, \\ University of California, Berkeley, CA 94720, USA \\ Y. KLUGER \\ Theoretical Division, Los Alamos National Laboratory, Los Alamos, NM 87545, \\ $U S A$

\begin{abstract}
Disoriented chiral condensates are manifested as long wavelength pionic oscillations and their interaction with the thermal environment can be a significant source of dileptons. We calculate the yield of such dilepton production within the linear sigma model and illustrate the basic features of the dilepton spectrum in a schematic model. We find that the dilepton yield with invariant mass near and below $2 m_{\pi}$ due to the soft pion modes can be up to two orders of magnitude larger than the corresponding equilibrium yield. We conclude with a discussion on how
\end{abstract} \\ this enhancement can be detected by present dilepton experiments.
}

\section{Introduction}

Recently much attention has been deveted the thenomenon of so called disoriented chiral condensates (DCC the formation of a DCC is that after the restoration of chiral symmetry in a given region of space-time the system may relax into a misaligned vacuum with a non-vanishing expectation value of the pion field. The system will then eventually decay into the normal vacuum by emitting soft and coherent pions. In the framework of ultrarelativistic heavy ion collisions apossible way to gen-

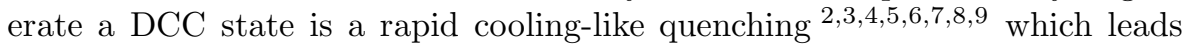
to a significant enhancement of low momentum pion modes. The resulting occupation numbers may then become large and lead to the emission of many pions in the same isospin state. In such an ideal scenario, the neutral pion fraction $f=<\pi_{0}>/<\pi>$ exhibits an anomalous distribution, $P(f)=1 / 2 \sqrt{f}$, 
which has been suggested as an experimental signal $10,11,22$. However, if several separate domains are formed (i.e. if the size of the system is large in comparison with the correlation length), as may well occur in heavy-ion collisions, the signal is correspondingly degraded and the distribution approaches its normal form, an approximately normal distribution centered around $f=\frac{1}{3}[3,14$. More advanced methods of analysis wuld then be needed, such as a the use of wavelets 15 or cumulative moments 16 .

The enhancement of low-momentum pion modes, which is associated with the formation of DCC states, should naturally lead to a strong enhancement in the dilepton channel. While the collective oscillation of the isgspin current leads to an appreciable signal only at very small invariant masse 17 , the annihilation of the low-momentum modes should result in a strong enhancement close to $M_{i n v} \simeq 2 m_{\pi}$. Moreover the signal should be confined to small momenta of the virtual photon since only the lowest momentum pion modes are enhanced. This enhancement will be the subject of my talk. It should be further noted that a similar enhancement has been predicted in the photon channel 18 . More details can be found in refle.

\section{Schematic picture of a DCC}

Let us start by defining what we mean by a quench and by convincing ourselves that such a quench indeed leads to an enhancement of low momentum pion modes. The basic idea of a quench is illustrated in Figure 11: Assume chiral symmetry has been restored, i.e. the system sits in middle of the effective potential, $\langle\sigma\rangle=0$. After rapid cooling it might happen that the system remains in the middle while the effective potential changes to a Mexican hat shape. This situation is highly unstable and the system wants to relax down into the rim of the Mexican hat. Since initially there is no prefered direction in isospin, the system may roll down in any direction, $\sigma, \pi_{1}, \pi_{2}, \pi_{3}$, where $\pi_{i}$ denotes the Cartesian components of the pion field. Thus large amplitudes of the pion field are generated before finally, due to explicit chiral symmetry breaking, the system eventually relaxes into the true vacuum, $\langle\sigma\rangle=f_{\pi}$, $<\pi>=0$.

In order to see that the low momentum modes are enhanced, let us look at the equations of motion of the pion field in the linear sigma model (here we ignore the effect of thermal fluctuation for simplicity).

$$
\left(\partial_{t}^{2}-\nabla^{2}\right) \vec{\Phi}+\lambda\left(\vec{\Phi}^{2}+\sigma^{2}-v^{2}\right) \vec{\Phi}=0
$$

with the general solution

$$
\Phi(t)=\Phi_{0} \mathrm{e}^{ \pm \mathrm{i} \omega_{\mathrm{k}} \mathrm{t}}
$$



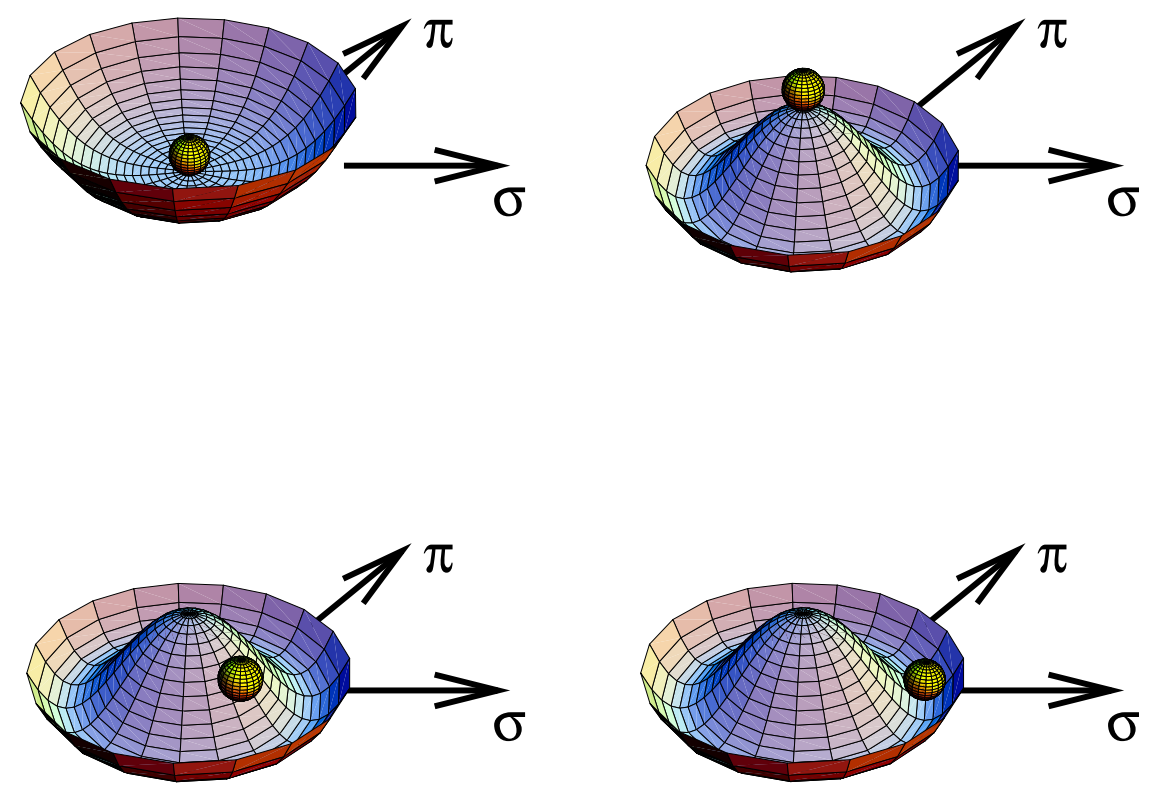

Figure 1: Schematic view of the quench scenario

where

$$
\omega_{k}=\sqrt{m_{\pi}^{* 2}+k^{2}} \text {, and } m_{\pi}^{* 2} \simeq \lambda\left(<\sigma>^{2}-v^{2}\right)
$$

In the ground state, $\langle\sigma\rangle=f_{\pi}$ and $m_{\pi}^{*}=m_{\pi}$. In the quench scenario, on the other hand, $\left\langle\sigma>\simeq 0\right.$ and $m_{\pi}^{* 2} \simeq-\lambda v^{2}<0$ and hence for momenta smaller than the absolute value of the pion mass, $k<\left|m_{\pi}^{*}\right|$, the frequency $\omega_{k}$ turns out to be imaginary and consequently the modes grow exponentially.

$$
\Phi(t) \simeq \Phi_{0} \mathrm{e}^{+\left|\omega_{\mathrm{k}}\right| \mathrm{t}}
$$

Thus, only the modes with $k<\left|m_{\pi}^{*}\right| \simeq 200 \mathrm{MeV}$ will be enhanced. The different orientations of the DCC fields in isospin space result from the the 
initial value of the pion field $\Phi_{0}$. Initially, before the quench occurs, $\Phi_{0}$ is more or less randomly oriented in isospin space. Therefore, depending on the direction $\Phi_{0}$ points at the time of the quench different isospin modes become amplified.

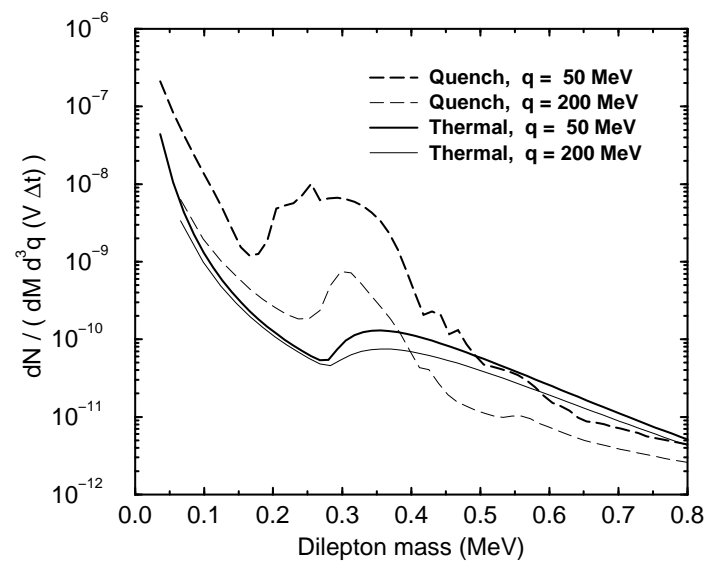

Figure 2: Dilepton invariant mass spectra for thermal (full lines) and quench (dashed lines) initial conditions. Shown are the spectra for two different values of the three dilepton three momentum $q$. The result has been obtained in the $1 / \mathrm{N}$ mean-field approximation to the linear sigma model.

\section{Dilepton production}

A detailed discussion on how to calculate the production of dileptons from the dynamics of the sigma model can be found in ref. 19 . We, therefore, restrict ourselves to the basic concepts and formulae. In general, in a non-equilibrium system, such as the dynamical evolution of DCC fields, the in and out states are not asymptotic states and the density matrices describing the system are not diagonal (except in isospin and charge). In this paper, we will neglect quantum effects caused by the off-diagonal matrices of the system in the calculation of the dilepton production. Therefore, the dilepton production yield can be given by the $S$ matrices of the electromagnetic transition between different states 22 ,

$$
\frac{d N_{\ell^{+} \ell^{-}}}{d^{4} q}=\frac{\alpha^{2}}{6 \pi^{3}} \frac{B}{q^{4}}\left(q^{\mu} q^{\nu}-q^{2} g^{\mu \nu}\right) W_{\mu \nu}(q)
$$




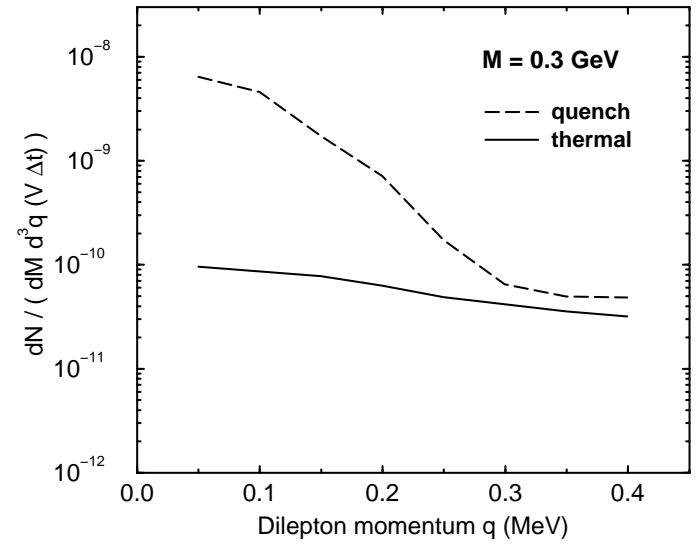

Figure 3: Momentum spectra for thermal (full lines) and quench (dashed lines) initial conditions for dilepton pairs of invariant mass $M=300 \mathrm{MeV}$. The result has been obtained in the $1 / \mathrm{N}$ mean-field approximation to the linear sigma model.

$$
W_{\mu \nu}(q)=\frac{1}{\mathcal{Z}} \int d^{4} x d^{4} y e^{-i q \cdot(x-y)} \operatorname{Tr}\left[\hat{\rho} \hat{j}_{\mu}(x) \hat{j}_{\nu}^{\dagger}(y)\right],
$$

where we have summed over the final states, $\mathcal{Z}=\operatorname{Tr}[\hat{\rho}]$, and

$$
B=\left(1-\frac{4 m_{\ell}^{2}}{q^{2}}\right)^{1 / 2}\left(1+\frac{2 m_{\ell}^{2}}{q^{2}}\right) .
$$

Since we are interested in the production of electron-positron pairs, we shall neglect the lepton mass $m_{\ell}$ in the following, implying $B=1$.

For the actual calculation, we have modified the temporal integration boundaries from the asymptotic times $t= \pm \infty$ to finite initial and final times $t_{i}$ and $t_{f}$. This restriction leads to artificial 'turn on / turn off' effects at low masses in the dilepton invariant mass spectrum as discussed in detail in 19 . The dynamical input provided by the time-dependent solution of the linear sigma model enters in eq. (5) via the current-current correlation function

$$
\operatorname{Tr}\left[\hat{\rho} \hat{j}_{\mu}(x) \hat{j}_{\nu}^{\dagger}(y)\right] .
$$

In the linear sigma model the electromagnetic current is given by

$$
\hat{j}_{\mu}(x)=\frac{i}{2}\left[\hat{\pi}^{\dagger}(x) \stackrel{\leftrightarrow}{\partial}_{\mu} \hat{\pi}(x)-\hat{\pi}(x) \stackrel{\leftrightarrow}{\partial}_{\mu} \hat{\pi}^{\dagger}(x)\right]=\hat{\pi}_{1}(x) \partial_{\mu} \hat{\pi}_{2}(y)-\hat{\pi}_{2}(x) \partial_{\mu} \hat{\pi}_{1}(y),
$$




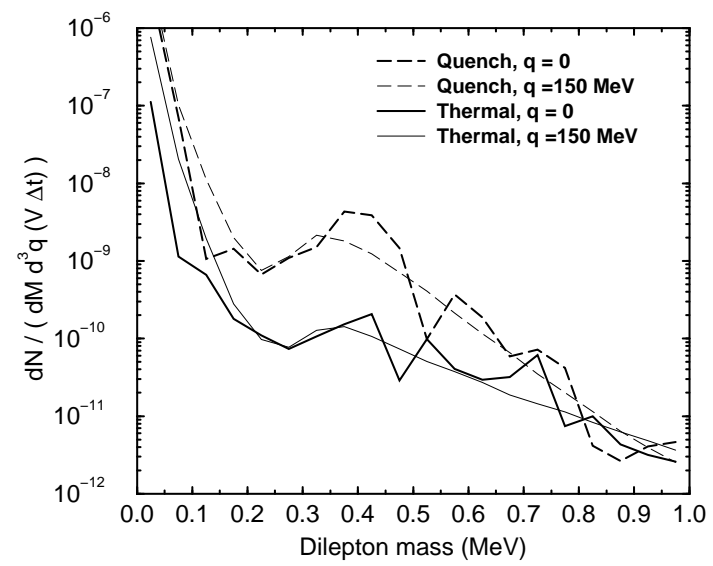

Figure 4: The invariant mass spectrum for both thermal initial conditions (solid curves) and the corresponding quench scenario (dashed curves), for either back-to-back dileptons having $\mathbf{q}=\mathbf{0}$ (heavy curves) and dileptons with a finite momentum of $q=150 \mathrm{MeV}$ (light curves). The result has been obtained in the semi-classical approximation to the linear sigma model.

where the complex charged pion field operators are related to the Cartesian components by

$$
\hat{\pi}(x)=\frac{1}{\sqrt{2}}\left[\hat{\pi}_{1}(x)+i \hat{\pi}_{2}(x)\right], \hat{\pi}^{\dagger}(x)=\frac{1}{\sqrt{2}}\left[\hat{\pi}_{1}(x)-i \hat{\pi}_{2}(x)\right] .
$$

We shall neglect the quadratic coupling in the gauged linear sigma model and the anomalous electromagnetic coupling of $\pi^{0}$, which all contribute to the dilepton production only to higher orders in the fine structure constant $\alpha=e^{2} / 4 \pi$.

We have calculated this correlation function in the $1 \not N$ mean-field approximation of the linear sigma model by Kluger et al. 120 as well as in the semi-classical treatment of Randrup 21 .

In both model calculations, mean-field as well as semi-classical, we have calculated the dilepton yield for two different initial conditions with the same energy density.

1. Thermal initial conditions. These initial conditions are such that the resulting system corresponds to a thermal ensemble of pions and sigmas. In this case we have verified that the resulting dilepton yield agrees with that of a pion gas. 


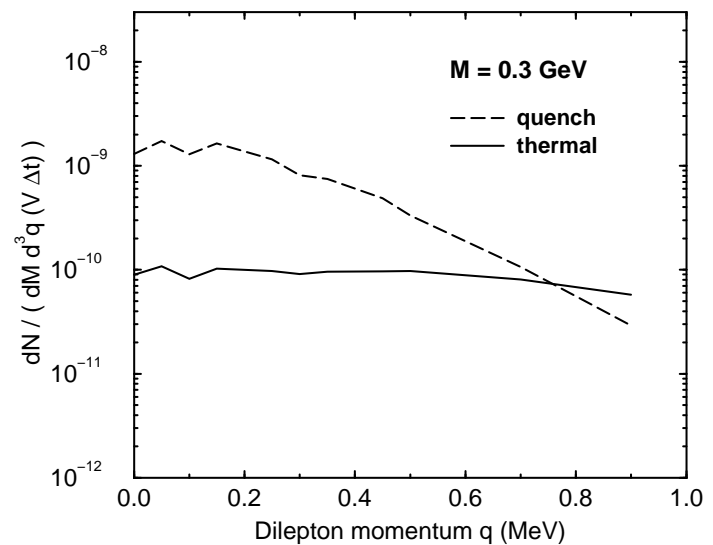

Figure 5: The production rate $\left(d^{4} N / d M d^{3} q(V \Delta t)\right)$ as a function of the magnitude of the dilepton momentum q, for dilepton masses near $M=300 \mathrm{MeV}$. The result has been obtained in the semi-classical approximation to the linear sigma model.

2. Quench initial conditions. In this case the system is initialized at small values for $\sigma$-field. Consequently a considerable fraction of the thermal energy is taken up by the potential (keeping the total energy density the same), leading to a cold initial configuration well inside the unstable region. As a result a well developed DCC will be temporarily formed until the system finally returns to thermal equilibrium.

In fig.2 we show the resulting dilepton invariant mass spectra for thermal and quench initial conditions for different three momenta of the virtual photon. Clearly a strong enhancement (factor $\simeq 100$ ) is seen around invariant masses of $\simeq 2 m_{\pi}$. This enhancement is confined to small momenta, as can be seen in fig. 3 where we plot the dilepton momentum spectrum for pairs having invariant mass $M=300 \mathrm{MeV}$. A similar behavior is found in the semi-classical calculation (see figures 1 and 5 ). Here the enhancement is somewhat smaller which is partially due to the additional mode mixing in the semi-classical treatment as well as the considerable larger grid size, which produces a faster damping of the low-momentum modes. 


\section{A schematic model}

With the mean-field and semi-classical treatments, the time-dependent fields are dynamically coupled to the quasi-particle modes and the soft and hard modes of the fields are treated on an equal footing. To understand the main features of dilepton production due to the soft modes, we shall now consider the dilepton production process in a schematic model in which the soft and hard modes are coupled only via the electromagnetic interaction causing dilepton production. The hard modes are then represented by a thermal gas of pions having a specified temperature $T$, while the soft modes will be evolved dynamically according to the one-dimensional Bjorken expansion scenario. Hence the density operator can be factorized, $\hat{\rho}=\hat{\rho}_{t h} \otimes \hat{\rho}_{D C C}$, where $\hat{\rho}_{t h}$ is the normalized density operator for the thermal gas and $\hat{\rho}_{D C C}$ is the normalized density operator for the soft modes, referred to as the DCC field.

The current-current correlator $\hat{W}_{\mu \nu}(q)$ can then be decomposed according to how many thermal pions are involved in the dilepton production process. The first term represents the coherent emission of dileptons from DCC fields alone and involves no thermal pions,

$$
W_{\mu \nu}^{(0)}(q)=\int d^{4} x d^{4} y\left\langle\hat{j}_{\mu}(x) \hat{j}_{\nu}^{\dagger}(y)\right\rangle_{D C C} \mathrm{e}^{-i q \cdot(x-y)}=\left\langle\hat{j}_{\mu}(q) \hat{j}_{\nu}^{\dagger}(q)\right\rangle_{D C C},
$$

where the DCC expectation value is $\langle\cdots\rangle_{D C C}=\operatorname{Tr}\left[\hat{\rho}_{D C C} \cdots\right]$.

Contributions to the current-current correlator from processes involving a thermal pion are

$$
\begin{aligned}
W_{\mu \nu}^{(1)}(q) & =\int \frac{d^{3} k}{2 \omega_{k}(2 \pi)^{3}}\left\{( 2 k _ { \mu } + q _ { \mu } ) ( 2 k _ { \nu } + q _ { \nu } ) \left[\left(1+n_{k}^{+}\right)\left\langle\hat{\pi}^{\dagger}(-q-k) \hat{\pi}(-q-k)\right\rangle_{D C C}\right.\right. \\
& \left.+\left(1+n_{k}^{-}\right)\left\langle\hat{\pi}(q+k) \hat{\pi}^{\dagger}(q+k)\right\rangle_{D C C}\right]+\left(2 k_{\mu}-q_{\mu}\right)\left(2 k_{\nu}-q_{\nu}\right) \\
& \left.\cdot\left[n_{k}^{+}\left\langle\hat{\pi}(q-k) \hat{\pi}^{\dagger}(q-k)\right\rangle_{D C C}+n_{k}^{-}\left\langle\hat{\pi}^{\dagger}(k-q) \hat{\pi}(k-q)\right\rangle_{D C C}\right]\right\}
\end{aligned}
$$

The first two terms in the above equation correspond to the emission of one pion together with a dilepton by the DCC field. The factor $1+n_{k}^{ \pm}$is a result of the Bose enhancement in the final state. The second two terms proportional to $n_{k}^{ \pm}$represent the annihilation or absorption of one thermal pion by the DCC field.

From Eq. (11) one can readily see how the momentum distribution of the DCC field is imprinted onto the dilepton spectrum. Once the momentum of the dilepton is large compared to the inverse of the DCC domain size, the integral no longer has support from the Fourier transform of the pion field $\left\langle\hat{\pi}^{\dagger}(k \pm q) \hat{\pi}(k \pm q)\right\rangle_{D C C}$, which restricts the contribution to low momenta and 
to a small window in invariant mass. For example, if one considers a classical field that oscillates with a typical frequency of $\omega \simeq m_{\pi}$ and has a Gaussian distribution of width $R_{\perp}$ in coordinate space, one can see from Eq. (11) that the dilepton yield will be concentrated around an invariant mass of $M \simeq 2 m_{\pi}$. The width of this distribution will be of the order of $1 / R_{\perp}$ in invariant mass as well as in the three-momentum $\boldsymbol{q}$.

Given this schematic model, it is possible to take approximate account of expansion by subjecting the DCC field to a bogst-invariant Bjorken expansion. For details of this calculation we refer to ref. 19 . The resulting dilepton invariant mass spectrum is shown in fig. 6 together with the corresponding average thermal rate.

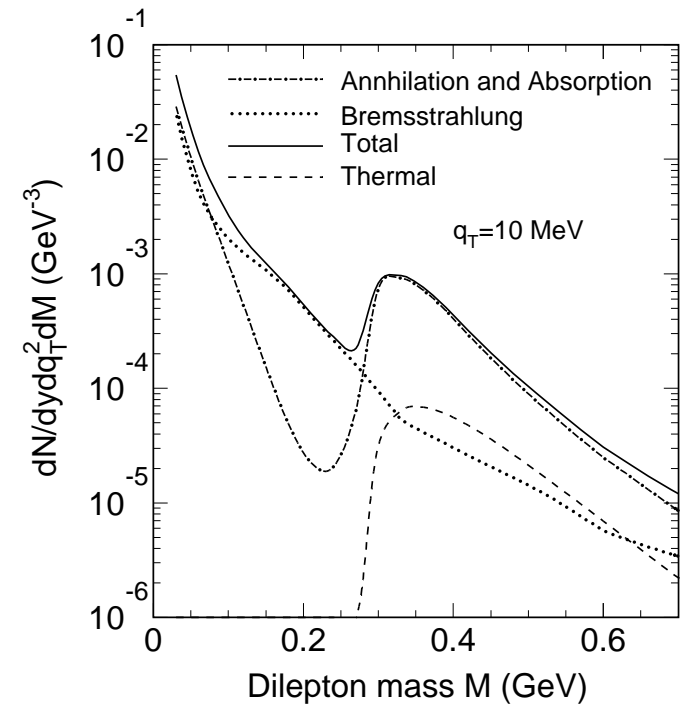

Figure 6: Dilepton spectrum from the DCC field, both bremsstrahlung (dotted), annihilation and absorption (dot-dashed), and their sum (solid), as well as the thermal emission (dashed). The initial temperature of the thermal environment is $T_{0}=145 \mathrm{MeV}$, while the characteristic energy density carried by the DCC field is $\epsilon_{0}=58 \mathrm{MeV} / \mathrm{fm}^{3}$.

First we find that the coherent emission rate is negligible as compared to the incoherent emission. Second, the incoherent dilepton emission is very significant in comparison with the thermal production. The spectrum from the pion annihilation and absorption by the DCC field (dot-dashed) has a structure manifest of two components in the second two terms in Eq. (11), depending 
on the energy flow. In the contribution from the annihilation with the DCC field, energy flows out of the DCC field, therefore the dilepton spectrum has a threshold at $M=2 m_{\pi}$, if the DCC pion field oscillate with a minimum frequency of $m_{\pi}$. If the energy flows into the DCC field, dileptons are then emitted when thermal pions are absorbed by the DCC field. The dilepton spectrum from these absorption processes has no threshold and dominates the annihilation and absorption spectrum at small invariant masses $M<m_{\pi}$, as seen in fig. 6.

Shown as the dotted line in fig. 6 is the dilepton spectrum due to pion bremsstrahlung from the DCC field. These bremsstrahlung contributions result from transitions between different modes of the DCC, which are a result of so called parametric resonances (for details see 19 ).

In fig. 6 is also shown the sum of the different contributions to dilepton spectrum from the DCC field (solid curve) as well as the contribution from annihilation in the cooling pion gas. In general, one can see that the incoherent dilepton production below and near $2 m_{\pi}$ threshold region is significantly larger than the thermal production. As we already pointed out, because of the finite spatial size of DCC field in the transverse direction, dileptons from the DCC field exhibit a much faster decrease with the transverse momentum $q_{\perp}$ than those due to thermal production.

\section{Observational aspects}

So far we have established that the presence of DCC states leads to a strong enhancement of the dilepton production at invariant masses close to $M \simeq 2 m_{\pi}$. We have also shown that this enhancement is confined to small momenta of the virtual photons $p<300 \mathrm{MeV}$. The question of course remains to which extent this rather unique signal can be observed in an actual experiment, where other, more conventional sources may dominate the dilepton measurement. For the mass range under consideration the Dalitz decay of the $\eta$ meson should be the most dominant background. Furthermore certain acceptance cuts need to be applied in order to minimize the combinatorial background from false pairs. In order to estimate these various backgrounds, we have used a recent transport calculation for dilepton production for $\mathrm{Pb}+\mathrm{Pb}$ collision at $156 \mathrm{AGeV} 24$. This calculation contains among others the $\eta$-Dalitz decay channel as well as pion annihilation. In order to calculate the contribution from the pion annihilation on DCC states we have extracted from the above mean-field calculation the ratio of quench to thermal dilepton production.

$$
R\left(M, p ; M_{\text {ref }}=350 \mathrm{MeV}\right)=\frac{Y_{\text {quench }}(M, p)}{Y_{\text {thermal }}\left(M_{\text {ref }}=350 \mathrm{MeV}, p\right)}
$$


where $Y_{\text {quench }}, Y_{\text {thermal }}$ stand for the dilepton yield for given $\mathrm{M}$ and p. $M_{\text {ref }}$ is the value of the invariant mass for which the matching between the two calculations is done for all $p_{t}$. Assuming, that the dilepton production in the heavy ion collisions is more or less thermal we can estimate the dilepton yield from $\mathrm{DCC}$ states in a $\mathrm{Pb}+\mathrm{Pb}$ collision by multiplying the dilepton yield from pion annihilation as obtained from the transport calculation with the above ratio (12)

$$
Y_{D C C}(M, p)=Y_{\text {transp. }}\left(M_{\text {ref }}=350 \mathrm{MeV}, p\right) R\left(M, p ; M_{\text {ref }}=350 \mathrm{MeV}\right)
$$

This of course ignores any non-thermal contribution as well as possible temperature dependences of the enhancement factor.

In fig. 7 we show the resulting dilepton spectra assuming perfect detector acceptance (i.e. no acceptance cuts). In the left panel we show the spectrum for all dileptons with transverse momentum smaller than $150 \mathrm{MeV}$. The right panel shows the dilepton mass spectrum for all transverse momenta. Clearly, the signal is at least as large as the competing $\eta$-Dalitz decay and thus should be observable especially if the statistics allow to look at dilepton momenta below $150 \mathrm{MeV}$.
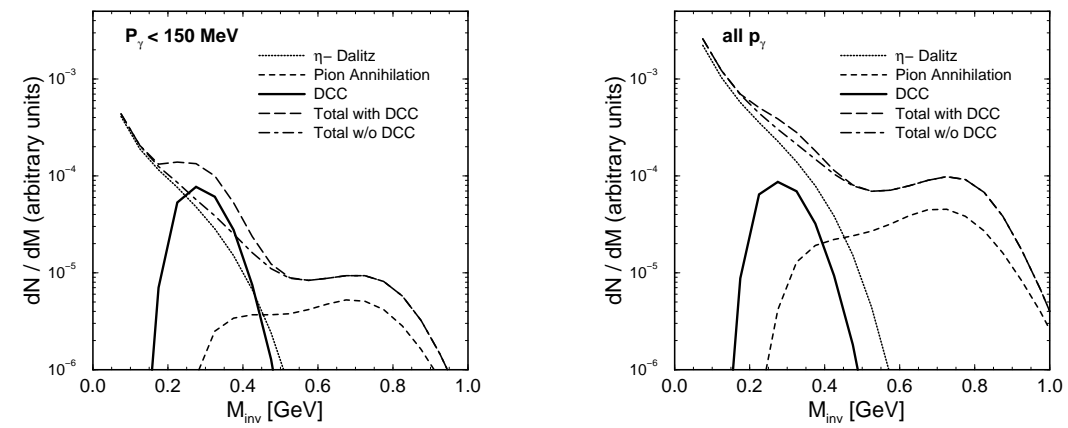

Figure 7: Dilepton invariant mass spectrum for $\mathrm{Pb}+\mathrm{Pb}$ collisions at $158 \mathrm{AGeV}$ as measured by a perfect detector. Shown are only the channel relomint for the discussion, but additional channel are taken into account in the total yield (see24).

In fig. 8 we have applied the cuts used by the CERES collaboration 23 . The most important cut is that on the transverse momentum of each individual lepton. Only pairs where each of the leptons has $p_{t}>200 \mathrm{MeV}$ are accepted. In this case, as it is clear from the figure, our proposed signal is not visible. 

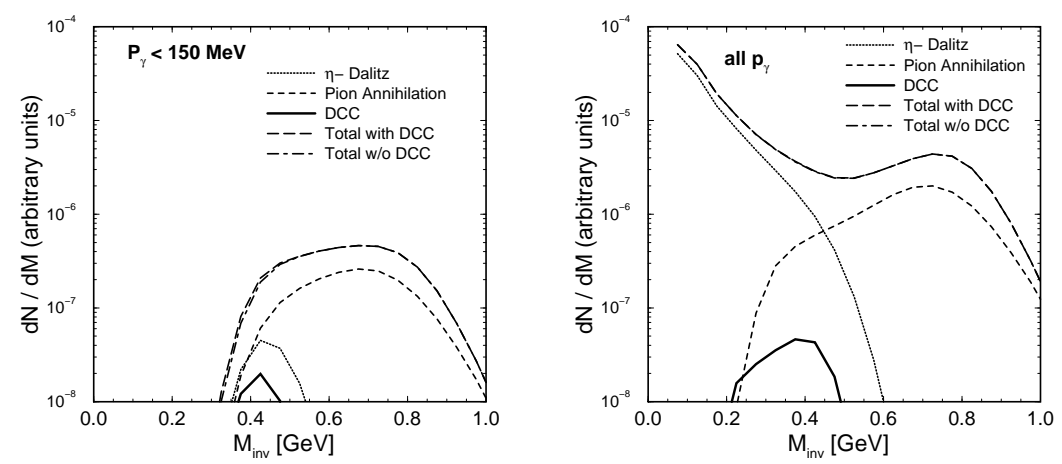

Figure 8: Same as Fig. 月, but now the CERES acceptance have been applied.

However, there might be a possibility that for small invariant masses CERES can relax the transverse momentum cut values as low as $60 \mathrm{MeV}$ 25. In this case the signal again should be visible as shown in fig. 9. Of course our estimate started from the assumption that in each event the conditions for a quench are satisfied. This might be somewhat optimistic. However, if the system equilibrates in the high temperature phase and generates sufficient rapid expansion this assumption should be fulfilled to a large extent. Furthermore, we have not included any dissipative processes which could destroy the DCC-states before the system has frozen out. Consequently if the lifetime of a DCC turns out to be considerably shorter than that of the system, our results have to be reduced accordingly.

\section{Summary}

We have calculated the production of dileptons from disoriented chiral condensates using a quantum mean-field as well as a semi-classical treatment for the time evolution in the linear sigma model. We have compared the dilepton spectra obtained when using so called quench initial conditions, which lead to a strong enhancement of long wave length pion modes (DCC), with those obtained from thermal initial conditions. Compared to the thermal spectrum the quench initial conditions lead to a strong enhancement (factor 20 - 100 depending on the model) at an invariant mass of about $M \simeq 2 m_{\pi}$. This enhancement is confined to dilepton momenta of $q \leq 300-500 \mathrm{MeV}$ and also 

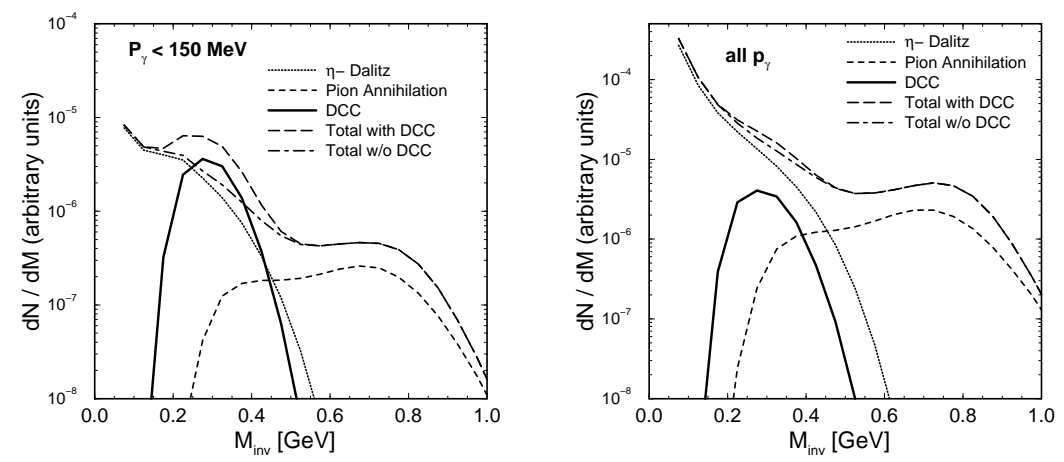

Figure 9: Same as Fig. 8, but the CERES momentum cuts have been reduced to $60 \mathrm{MeV}$.

rather narrow in invariant mass. Within a schematic model, we could also address the more realistic scenario of a longitudinal expansion. We find that the above enhancement remains also in this case.

Both dynamical solutions, which are based on distinct approximations to the linear sigma model, predict qualitatively the same enhancement: A large bump at $M \simeq 2 m_{\pi}$ as well as an enhancement at low invariant masses. Overall, the quantum mean-field model seems to yield a larger enhancement than the semi-classical treatment. This quantitative difference may be due to several factors. First, the Bose enhancement factors are absent in the semi-classical treatment. Second, the semi-classical treatment incorporates the mode mixing resulting from the non-linear interaction and this mechanism tends to reduce the number of pions in the DCC state. Both of these features lead to a somewhat smaller signal.

As far as experimental observation of this enhancement is concerned, we have carried out a rough estimate of the expected signal for a $\mathrm{Pb}+\mathrm{Pb}$ collision at SPS energies. We have shown that the signal would be strong enough to stand out from the main background, the Dalitz decay of the $\eta$-meson. We furthermore have applied the acceptance cuts of the CERES experiment. With the present cuts of $p_{t}>200 \mathrm{MeV}$ the signal would not be visible by the CERES detector. However, if these cuts can be relaxed to $p_{t}>60 \mathrm{MeV}$, the signal should be observable, provided, of course that these DCC states are ever being formed in SPS-energy heavy ion collisions. 


\section{Acknowledgments}

This work was supported by the Director, Office of Energy Research, Office of High Energy and Nuclear Physics, Divisions of High Energy Physics and Nuclear Physics of the U.S. Department of Energy under Contract No. DEAC03-76SF00098.

1. J.D. Bjorken, K.L. Kowalski, and C.C. Taylor, SLAC preprint SLACPUB-6109, Proc. of Les Rencontres de la Vallée D' Aoste, La Thuile, 1993, ed. M. Greco, Editions Frontier, p. 507 (1993).

2. K. Rajagopal and F. Wilczek, Nucl. Phys. B404, 577 (1993).

3. S. Gavin, A. Goksch, and R.D. Pisarski, Phys. Rev. Lett. 72 2143, (1994).

4. S. Gavin and B. Müller, Phys. Lett. B329, 486 (1994).

5. M. Asakawa, Z. Huang, and X.-N. Wang Phys. Rev. Lett. 74, 3126 (1995).

6. D. Boyanovsky, H.J. de Vega, and R. Holman, Phys. Rev. D 51, 734 (1995).

7. F. Cooper, Y. Kluger, and E. Mottola, and J.P. Paz, Phys. Rev. D51, 2377 (1995).

8. Y. Kluger, F. Cooper, E. Mottola, Phys. Rev. C54, 3298 (1996).

9. J. Randrup, Phys. Rev. Lett. 77, 1226 (1996).

10. A.A. Anselm and M.G. Ryskin, Phys. Lett. B266, 482 (1991).

11. J.-P. Blaizot and A. Krzywcki, Phys. Rev. D46, 246 (1992).

12. J.D. Bjorken, Acta Phys. Pol. B23, 561 (1992).

13. R.D. Amado and Y. Lu, Phys. Rev. D54, 7075 (1996).

14. J. Randrup, Nucl. Phys. A616, 531 (1997).

15. Z. Huang, I. Sarcevic, R. Thews, and X.-N. Wang, Phys. Rev. D54, 750 (1996); J. Randrup and R. Thews, Phys. Rev D97, 4392 (1997).

16. T.C. Brooks, et al., MiniMax Collaboration, hep-ph/9609375, 1996.

17. Z. Huang and X.-N. Wang, Phys. Lett. B383, 457 (1996).

18. D. Boyanovsky, H.J. de Vega, R. Holman, and S. Prem Kumar, hepph/9701360 and hep-ph/9703422.

19. Y. Kluger, V. Koch, J. Randrup, and X.N. Wang, Phys. Rev. C, in print, nucl-th/970418

20. Y. Kluger, J. M. Eisenberg, B. Svetitsky, F. Cooper, and E. Mottola, Phys. Rev. D45, 4659 (1992).

21. J. Randrup, Phys. Rev. D55, 1188 (1997).

22. L.D. McLerran and T. Toimela, Phys. Rev. D31, 545 (1985).

23. G. Agakichiev et al., Phys. Rev. Lett. 75, 1272 (1995).

24. V. Koch and C. Song, Phys. Rev. C54 1903 (1996). 
25. A. Dress, private communication. 